Pacific Journal of Mathematics

A FUNCTIONAL INDEPENDENCE THEOREM FOR SQUARE 


\title{
A FUNCTIONAL INDEPENDENCE THEOREM FOR SQUARE MATRICES
}

\author{
C. W. Mendel And I. A. BarnetT
}

The purpose of this paper is to prove the following independence theorem:

If $A$ is a square matrix of order $n$, the Jacobian of the $n$ traces of $A, A^{2}, \cdots, A^{n}$ with respect to each set of $n$ distinct elements of $A$, at least one of which is a diagonal element, is never identically zero in the $n^{2}$ elements of $A$.

The problem arose originally in connection with a certain system of differential equations of the second order [1]. This led to the investigation of the properties of a class of determinants which are generalizations of the classical determinant of Vandermonde [2]. The latter half of [2] includes a proof of the independence theorem as given by Perron who used mathematical induction. We now give the proof first devised by the authors in 1940. It is interesting for two reasons; first, new results in the algebra of matrices are brought to light and second, matrices are constructed for which the $n$ traces are independent.

1. Notations and terminology. Let $A=\left(a_{i j}\right)$ be a square matrix of order $n$ whose elements are independent indeterminates over an arbitrary field. Let $a_{i j}^{(m)}$ stand for the element in the $i$ th row and $j$ th column of the $m$ th power of $A$. The determinant

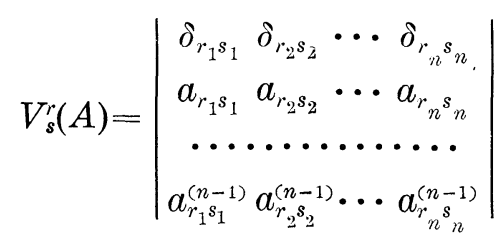

where $r_{1}, \cdots, r_{n}$ and $s_{1}, \cdots, s_{n}$ are arbitrary integers in the range 1 to $n$, equal or unequal, and $\delta_{i j}$ is the Kronecker delta, is called a generalized determinant of Vandermonde. It reduces to the classical determinant of Vandermonde if $A$ is a diagonal matrix.

Consider any set $S$ of $n$ distinct elements $a_{s_{1} r_{1}}, a_{s_{2} r_{2}}, \cdots, a_{s_{n} r_{n}}$ of the matrix $A$. Also consider the set $T$ of $n$ traces $t_{1}, t_{2}, \cdots, t_{n}$ of $A$, $A^{2}, \cdots, A^{n}$. Let us represent by $\partial T / \partial S$ the Jacobian of the set $T$ with

Received January 28, 1955, and in revised form September 21, 1956. 
respect to the set $S$, so that equation (13) of [2] may be written $\partial T / \partial S$ $=n ! V_{s}^{r}(A)$.

We have already introduced the two ordered sets of indices $r=\left(r_{1}\right.$, $\left.r_{2}, \cdots, r_{n}\right)$ and $s=\left(s_{1}, s_{2}, \cdots, s_{n}\right)$, each on the range $1,2, \cdots, n$. When $r$ and $s$ are considered together, we shall speak of the dual-set $(r ; s)$ and we write this as a 2 by $n$ matrix:

$$
(r ; s)=\left(\begin{array}{c}
r_{1} r_{2} \cdots r_{n} \\
s_{1} s_{2} \cdots s_{n}
\end{array}\right) .
$$

The fact that $S$ consists of $n$ distinct elements may be stated by saying that no two columns of $(r ; s)$ are identical. We shall be interested in sets $S$ which, in addition to consisting of $n$ distinct elements, contain at least one diagonal element of $A$. Such sets will be called nonsingular, while all others will be called singular. The dual-set $(r ; s)$ corresponding to $S$ will be called nonsingular or singular according as $s$ is nonsingular or singular. Thus for a nonsingular $(r ; s)$ no two columns may be identical and in at least one column the indices in the first and second rows must be equal. Such a column will be called a diagonal column.

In this connection it will be convenient to introduce notations for two operators on sets of $n$ indices. Let $\alpha$ and $\beta$ be two distinct integers in the range $1,2, \cdots, n$, and let $a=\left(a_{1}, a_{2}, \cdots, a_{n}\right)$ be a set of $n$ (not necessarily distinct) integers in the same range. Then by

$$
(\alpha \beta) a=(\alpha \beta)\left(a_{1}, a_{2}, \cdots, a_{n}\right)
$$

we mean the set of integers $\left(a_{1}^{\prime}, a_{2}^{\prime}, \cdots, a_{n}^{\prime}\right)$ obtained from $\alpha$ by interchanging $\alpha$ and $\beta$.

In analogous fashion, by the notation

$$
[\alpha \beta] a=[\alpha \beta]\left(a_{1}, a_{2}, \cdots, a_{n}\right)
$$

we mean the set $\left(a_{1}^{\prime}, a_{2}^{\prime}, \cdots, a_{n}^{\prime}\right)$ obtained from $a$ by changing $\alpha$ to $\beta$ wherever $\alpha$ occurs in $a$. If $a^{\prime}=(\alpha \beta) a$, then $a=(\alpha \beta) a^{\prime}$ but, if $a^{\prime}=[\alpha \beta] a$, it is not always true that $a=[\alpha \beta] \alpha^{\prime}$. In fact, having passed from any set $a$ to another set $a^{\prime}$ by this latter method, it may not be possible to get back from $a^{\prime}$ to $a$ by using any such operators.

For brevity we write $\left(r^{\prime} ; s^{\prime}\right)=(\alpha \beta)(r ; s)$ to mean $r^{\prime}=(\alpha \beta) r, s^{\prime}=(\alpha \beta) s$. However, it will be convenient to attach a slightly different meaning to the operator $[\alpha \beta]$ when applied to a dual-set. In fact, $\left[r^{\prime} ; s^{\prime}\right]=$ $[\alpha \beta](r ; s)$ shall mean that $r^{\prime}=[\alpha \beta] r, s^{\prime}=[\beta \alpha] s$.

A nonsingular $(r ; s)$ (and the corresponding $S$ ) will be called unitary, if it contains exactly one diagonal column.

Finally, $(r ; s)$ (and the corresponding $S$ ) will be called proper if, when the diagonal columns are deleted from the set, the resulting two 
rowed matrix has no index common to both rows.

2. Outline of the proof. In the first step we prove the theorem for the case in which $S$ consists of the $n$ diagonal elements of $A$. In the remainder of the proof this case will then be excluded.

We next prove two lemmas:

LEMMA 1. If there exists a nonsingular set $S$ for which $\partial T / \partial S \equiv 0$ then there exists a unitary set $S^{\prime}$ for which $\partial T / \partial S^{\prime} \equiv 0$.

LEMмa 2. If there exists a unitary set $S$ for which $\partial T / \partial S \equiv 0$, then there exists a proper unitary set $S^{\prime}$ for which $\partial T / \partial S^{\prime} \equiv 0$.

The above lemmas reduce the problem to that of proving the theorem for all proper unitary sets. The method of proof of the lemmas enables us to restrict further the class of proper unitary sets for which the theorem need be proved. For each of the sets $S^{\prime \prime}$ in the sub-class we exhibit a matrix $A$ for which $\partial T / \partial S^{\prime \prime} \neq 0$. This, then, will complete the proof for all nonsingular $S$.

3. Reduction theorems. We now state five theorems called reduction theorems to be used in proving the two lemmas just stated. The conclusions in all five theorems are almost identical, the only variation being an implication in Theorem 4 while an equivalence is found in the other theorems ${ }^{1}$.

THEOREM 1. If $r^{\prime}=s$ and $s^{\prime}=r$ then $V_{s}^{r}(A) \equiv 0$ is equivalent to $V_{s^{\prime}}^{r^{\prime}}(A) \equiv 0$.

THEOREM 2. If $\left(r^{\prime} ; s^{\prime}\right)$ can be obtained from $(r ; s)$ by permuting the columns of the latter, then $V_{s}^{r}(A) \equiv 0$ is equivalent to $V_{s^{\prime}}^{r^{\prime}}(A) \equiv 0$.

THEOREM 3. If $\left(r^{\prime} ; s^{\prime}\right)=(\alpha \beta)(r ; s)$ then $V_{s}^{r}(A) \equiv 0$ is equivalent to $V_{s^{\prime}}^{r^{\prime}}(A) \equiv 0$.

Theorem 4. If $\left(r^{\prime} ; s^{\prime}\right)=[\alpha \beta](r ; s)$ then $V_{s}^{r}(A) \equiv 0$ implies that $V_{s^{\prime}}^{r^{\prime}}(A) \equiv 0$.

THEOREM 5. If, from a unitary $(r ; s)$ we derive $\left(r^{\prime} ; s^{\prime}\right)$ by changing the diagonal column of $(r ; s)$ to some new diagonal column, leaving

1 The reader will readily observe that, in some of these theorems, we have stated far less than could be said, but we have given them in the form in which they are actually used below. 
all other columns unaltered, then $V_{s}^{r}(A) \equiv 0$ is equivalent to $V_{s^{\prime}}^{r^{\prime}}(A) \equiv 0$.

All identities in these theorems are in the $n^{2}$ variables $a_{i j}$ of $A$. This will be understood to apply to all identities in which the variables are not explicitly stated.

4. Proofs of the reduction theorems. In Theorem 1 the fact that the equation $V_{s}^{r}(A) \equiv 0$ is an identity, permits us to replace $A$ by any other matrix; in particular by $A^{\prime}$, the transpose of $A$, and the equation is still true, so that $V_{s}^{r}\left(A^{\prime}\right) \equiv 0$. Since by hypothesis $r^{\prime}=s$ and $s^{\prime}=r$ we have:

$$
V_{s}^{r}\left(A^{\prime}\right) \equiv V_{r}^{s}(A) \equiv V_{s^{\prime}}^{r^{\prime}}(A) .
$$

In Theorem 2 we have at once $V_{s}^{r}(A) \equiv \pm V_{s^{\prime}}^{r^{\prime}}(A)$, since, except for order, the columns of the two determinants are the same.

For the proof of Theorem 3, let $p$ denote the transposition $(\alpha \beta)$ and $p j$ the image of $j$ under $p$. Let $B$ be the matrix defined by $b_{i j}=$ $a_{p i p j}$. Then it follows by induction on $k$ that $b_{i j}^{(k)}=a_{p i p j}^{(k)}$. Thus if $\left(r^{\prime} ; s^{\prime}\right)$ $=(\alpha \beta)(r ; s)$, we have $V_{s}^{r}(B) \equiv V_{s^{\prime}}^{r^{\prime}}(A)$ and the proof is complete.

For the proof of the Theorem 4, let the matrix $B$ have elements $b_{i j}^{(1)}$ defined by

$$
b_{i j}^{(k)}=a_{i j}^{(k)}+\lambda\left(a_{i \alpha}^{(k)} \delta_{j \beta}-a_{\beta j}^{(k)} \delta_{i \alpha}\right)-\lambda^{2} a_{\beta \alpha}^{(k)} \delta_{i \alpha} \delta_{i \beta},
$$

with $k=1$ and $\lambda$ an arbitrary parameter. Then it may be verified that (2) is correct for all $k$. Now if $V_{s}^{r}(A) \equiv 0$ we see that $V_{s}^{r}(B) \equiv 0$, the last expression being an identity not only in the $n^{2}$ elements $a_{i j}$ but also in $\lambda$. We shall show that the coefficient of the highest power of $\lambda$ in $V_{s}^{r}(B)$ is the desired $V_{s^{\prime}}^{r^{\prime}}(A)$ so that this determinant must vanish.

We note from the equation (2) that each element $b_{r_{j} s_{j}}, b_{r_{j} j_{j}}^{(2)}, \cdots$, $b_{r_{j^{s} j}}^{(n-1)}$ in the $j$ th column of $V_{s}^{r}(B)$ is of degree $m_{j}=\delta_{\alpha r_{j}}+\delta_{\beta s_{j}}$ in $\lambda$. Hence, every term of $V_{s}^{r}(B)$ is of degree $N=\sum_{j=1}^{n} m_{j}$ in $\lambda$. Now the coefficient of $\lambda^{m_{j}}$ in $b_{r_{j^{j} j}}^{(i-1)}$ is seen to be $\pm a_{r_{j} j_{j}}^{(i-1)}$ where $r_{j}^{\prime}=[\alpha \beta] r_{j}$ and $s_{j}^{\prime}=[\beta \alpha] s_{j}$, so that $\left(r^{\prime} ; s^{\prime}\right)=[\alpha \beta](r ; s)$. Hence the coefficient of $\lambda^{N}$ in $V_{s}^{r}(B)$ is $\pm V_{s^{\prime}}^{r^{\prime}}(A)$. This completes the proof of Theorem 4 .

Theorem 5 is obvious since we have $V_{s^{\prime}}^{r^{\prime}}(A) \equiv V_{s}^{r}(A)$.

5. Use of the reduction theorems. It is easy to verify that, if $(r ; s)$ is nonsingular, the application of any one of the reduction Theorems $1,2,3$ or 5 always leads to a nonsingular $\left(r^{\prime} ; s^{\prime}\right)$. This is not true for Theorem 4. In order to avoid reductions which lead to singular sets, we shall investigate the effect of Theorem 4 in detail.

By mates in $s$ of an index $\alpha$ which appears in $r$, we mean all in- 
dices $s_{i}$ for which $r_{i}=\alpha$. Each such index $s_{i}$ (and clearly, these will all be distinct for a nonsingular dual-set) will be called a mate in $s$ of $\alpha$. Similar definitions apply for mates in $r$.

We call two indices $\alpha$ and $\beta$ associates in $r$ if there is an index $\gamma$ in $s$ such that both $\alpha$ and $\beta$ are mates in $r$ of $\gamma$. Each of the numbers $\alpha, \beta$ will be called an associate in $r$ of the other. Similar definitions apply for associates in $s$.

We now consider $\left(r^{\prime} ; s^{\prime}\right)$ related to the nonsingular $(r ; s)$ by $\left(r^{\prime} ; s^{\prime}\right)$ $=[\alpha \beta](r ; s)$. According to the definition given in $\S 1,\left(r^{\prime} ; s^{\prime}\right)$ will be singular if the corresponding set of elements of $A$, viz., $a_{r_{1}^{\prime} s_{z_{2}}}, a_{r_{r_{2}} s_{z_{2}}}$, $\cdots, a_{r_{n}}{ }^{\prime} s_{n}$, does not contain a diagonal element of $A$, or if it does not consist of $n$ distinct elements of $A$. After some analysis we may show that this set will be singular if and only if, the numbers $\alpha$ and $\beta$ are such that one or more of the following is true:

I. The set $S$ corresponding to $(r ; s)$ contains exactly one diagonal element of $A$, and this one is either $a_{\alpha \alpha}$ or $a_{\beta \beta}$.

II. The numbers $\alpha, \beta$ are associates either in $r$ or in $s$.

III. The set $S$ corresponding to $(r ; s)$ contains either the two elements $a_{\alpha \alpha}$ and $a_{\beta \beta}$ or the two elements $a_{\alpha \beta}$ and $a_{\beta \alpha}$.

In applying Theorem 4 we avoid using numbers $\alpha, \beta$ satisfying any one of these conditions. It should be noted, however, that condition I constitutes no real restriction on the numbers $\alpha, \beta$ in case $n>2$ for, we may firstly apply Theorem 5 to carry the lone diagonal element into some element other than $a_{\alpha \alpha}$ or $a_{\beta \beta}$ and then, after applying Theorem 4, use Theorem 5 to restore the index of the diagonal element to its former value.

If, for a given pair of distinct integers $\alpha, \beta$ the operation $[\alpha \beta]$ carries a nonsingular $(r ; s)$ into a singular set, we shall call the pair $\alpha, \beta$ a blocked pair for $(r ; s)$.

6. The case of $n$ diagonal elements in $S$. In order to prove the Independence Theorem in the case $S\left(a_{11}, a_{22}, \cdots, a_{n n}\right)$ we may take $A$ to be the general diagonal matrix. In this case $\partial T / \partial S$ becomes the classical determinant of Vandermonde and is, of course, not identically zero. In the future we exclude this case.

7. Proof of Lemma 1. We now consider a nonsingular $(r ; s)$ containing more than one but less than $n$ diagonal columns and we prove that, by the use of Theorem 4 , such a set can be reduced to a nonsingular dual-set having exactly one diagonal column (that is, to a unitary set).

Consider the nonsingular $(r ; s)$ having exactly $k$ diagonal columns 
with $1<k<n$. By Theorems 2 and 3 we are justified in supposing that

$$
\begin{array}{lr}
r_{i}=s_{i}=1 & (i=1,2, \cdots, k), \\
r_{i} \neq s_{i} & (i=k+1, k+2, \cdots, n) .
\end{array}
$$

We need only show that a nonsingular dual-set having one less diagonal column can be obtained from this one.

Consider the following reductions:

I. If $\beta$ is such that $k<\beta \leqq n$ and $\beta$ does not occur in $(r ; s)$ let $\left(r^{\prime} ; s^{\prime}\right)=[k \beta](r ; s)$.

II. If $\alpha$ is such that $1 \leqq \alpha \leqq k$ and $\alpha$ does not occur in the last $n-k$ columns of $(r ; s)$, let $\left(r^{\prime} ; s^{\prime}\right)=[\alpha n](r ; s]$.

If either of these reductions is possible (that is, if there exists such an integer $\beta$ or such an integer $\alpha$ ), then the resulting $\left(r^{\prime} ; s^{\prime}\right)$ is nonsingular and has $k-1$ diagonal columns.

If neither applies, then each index $1,2,3, \cdots, n$ must occur at least once in the last $n-k$ columns of the set $\left(r^{*} ; s\right)$. Let us fix our attention on dual-sets for which this is true. Now, considering for the moment only the $2(n-k)$ indices which make up the last $n-k$ columns of $(r ; s)$, and bearing in mind that every integer $1,2, \cdots, n$ occurs at least once in these columns, let

$\lambda_{1}$ distinct integers $1,2, \cdots, k$ occur in $r$ alone,

$\lambda_{2}$ distinct integers $1,2, \cdots, k$ occur in $s$ alone, $k-\lambda_{1}-\lambda_{2}$ distinct integers $1,2, \cdots, k$ occur in both $r$ and $s$,

$\mu_{1}$ distinct integers $k+1, \cdots, n$ occur in $r$ alone,

$\mu_{2}$ distinct integers $k+1, \cdots, n$ occur in $s$ alone, $n-k-\mu_{1}-\mu_{2}$ distinct integers $k+1, \cdots, n$ occur in both $r$ and $s$. These numbers $\lambda_{1}, \lambda_{2}, \mu_{1}, \mu_{2}$ are restricted by the following inequalities :

$$
\begin{gathered}
0 \leqq \lambda_{1}, \quad 0 \leqq \lambda_{2}, \quad \lambda_{1}+\lambda_{2} \leqq k \\
\mu_{1} \geqq k-\lambda_{1}, \quad \mu_{2} \geqq k-\lambda_{2}, \quad \mu_{1}+\mu_{2} \geqq k .
\end{gathered}
$$

The last of these is a consequence of the three which precede it. The fifth is obtained by noting that the total number of distinct integers which occur in the last $n-k$ columns of $r$ must be less than or equal to $n-k$. The fourth is obtained in a similar fashion.

Consider now the reduction: 
III. If $\beta_{1}, \beta_{2}$ are such that $k<\beta_{1}<\beta_{2} \leqq n$ and if $\beta_{1}, \beta_{2}$ are both in $r$ alone or both in $s$ alone, but are not associates, let $\left(r^{\prime} ; s^{\prime}\right)=\left[\beta_{1} \beta_{2}\right](r ; s)$.

If III is applicable, the resulting $\left(r^{\prime} ; s^{\prime}\right)$ is nonsingular and we may apply I to reduce the number of diagonal columns.

If III is not applicable, then each pair of the $\mu_{1}$ numbers $k+1, \cdots$, $n$ which occurs in $r$ alone must be associates in $r$, and each pair of the $\mu_{2}$ numbers $k+1, \cdots, n$ which occurs is $s$ alone must be associates in $s$. These conditions require a minimum of $\mu_{2}-1$ repetitions ${ }^{2}$ in $r$ and $\mu_{1}-1$ repetitions in $s$, of some of the distinct numbers which occur there. Now, counting distinct integers and known repetitions in $r$ and $s$, we have

$$
\begin{aligned}
& \lambda_{1}+\left(k-\lambda_{1}-\lambda_{2}\right)+\mu_{1}+\left(n-k-\mu_{1}-\mu_{2}\right)+\mu_{2}-1 \leqq n-k, \\
& \lambda_{2}+\left(k-\lambda_{1}-\lambda_{2}\right)+\mu_{2}+\left(n-k-\mu_{1}-\mu_{2}\right)+\mu_{1}-1 \leqq n-k,
\end{aligned}
$$

from which we get

$$
k-1 \leqq \lambda_{2}, \quad k-1 \leqq \lambda_{1},
$$

respectively. Adding these two inequalities and using the fact that $\lambda_{1}$ $+\lambda_{2} \leqq k, k>1$, we readily find that $k=2, \lambda_{1}=\lambda_{2}=1$. Hence, for all dual-sets except these, the reductions I, II, III suffice to reduce the number of diagonal columns by one.

We must now deal with those sets for which reductions I, II, III do not apply. As we have just seen, such a set must have exactly two diagonal columns and it must have $\lambda_{1}=\lambda_{2}=1$. Thus, if the diagonal columns are $r_{1}=s_{1}=1, r_{2}=s_{2}=2$, then we may suppose that in the last $n-2$ columns of $(r ; s), 1$ occurs in $r$ alone and 2 occurs in $s$ alone.

For this set consider the reduction:

IV. If, after deleting diagonal columns, $\alpha$ and $\beta$ occur in $r$ alone (or in $s$ alone) with $1 \leqq \alpha \leqq 2,3 \leqq \beta \leqq n$ and $\alpha, \beta$ are not associates, then let $\left(r^{\prime} ; s^{\prime}\right)=[\alpha \beta](r ; s)$

If IV is applicable then the resulting set in nonsingular and has one less diagonal element. We shall prove that IV is always applicable to those sets for which I, II, III do not suffice. For the last $n-2$ columns of these sets we have supposed $\alpha=1$ occurs in $r$ alone and that there are $\mu_{1}$ numbers $\beta(3 \leqq \beta \leqq n)$ occurring in $r$ alone. If IV is not applicable there must be a minimum of $\mu_{1}$ repetitions in $s$ in order that 1 may be an associate of each of the $\mu_{1} \beta^{\prime} s$ occurring in $r$ alone. Thus in the last $n-2$ columns of $s$ there appear

2 If an integer occurs $k$ times in a set, we shall say that there are $k-1$ repetitions of this integer. We shall have occasion to refer to the total number of repetitions in a set without mentioning which integers are repeated. Thus if a set of $n$ integers contains exactly $\lambda$ distinct integers, there are $n-\lambda$ repetitions. 
(a) the number 2,

(b) $n-2-\mu_{1}$ of the numbers $3,4, \cdots, n$,

(c) $\mu_{1}$ repetitions of numbers in (a) or (b) .

This is a total of $n-1$ numbers occurring in $n-2$ columns, which is impossible. Thus, by means of reductions I, II, III, IV it is always possible to reduce $(r ; s)$ to a unitary set.

8. Proof of Lemma 2. We consider now only unitary dual-sets. Let us delete from $(r ; s)$ the diagonal column and then denote by $\lambda(r)$ the number of distinct indices in $r$, by $\lambda(s)$ the number of distinct indices in $s$, and by $\lambda(r, s)$ the number of distinct indices each of which occurs in both rows. It is clear that the number of distinct integers which occur among these $2(n-1)$ indices is $\lambda(r)+\lambda(s)-\lambda(r, s)$.

It is seen that if we go from one unitary dual-set to another by the use of the reduction Theorems 2,3 , or 5 , then $\lambda(r), \lambda(s), \lambda(r, s)$ remain unchanged. If reduction Theorem 1 is used, we have

$$
\lambda\left(r^{\prime}\right)=\lambda(s), \quad \lambda\left(s^{\prime}\right)=\lambda(r), \quad \lambda\left(r^{\prime}, s^{\prime}\right)=\lambda(r, s) .
$$

However, if reduction Theorem 4 is used to go from $(r ; s)$ to $\left(r^{\prime} ; s^{\prime}\right)$, we obtain the inequalities

$$
\lambda\left(r^{\prime}\right) \leqq \lambda(r), \quad \lambda\left(s^{\prime}\right) \leqq \lambda(s), \quad \lambda\left(r^{\prime}, s^{\prime}\right) \leqq \lambda(r, s) .
$$

We now show that we can always change a unitary set to a proper unitary set (that is, one for which $\lambda(r, s)=0$ ) by means of the reduction theorems. In this connection we should recall that, while application of Theorem 4 for a pair of integers $\alpha, \beta$ would lead to a singular set if the diagonal column of $(r ; s)$ has index $\alpha$ or $\beta$, this is no real restriction since, when $n>2$ we can first apply Theorem 5 to change the index of the diagonal column. Since, by Theorem 2, the diagonal column may be made the first column so that $r_{1}=s_{1}$, we now let $r_{1}=s_{1}$ $=x$ where $x$ is merely a symbol for the index of the diagonal column whose value we shall not specify.

Let us now suppose that a unitary set $(r ; s)$ is such that $\lambda(r, s)$ has been reduced as far as possible by means of Theorem 4 . We wish to show that in this case $\lambda(r, s)=0$. Suppose $\lambda(r, s)>0$. If there were an index (in the range $1,2, \cdots, n)$ which did not occur in $(r ; s)$, then Theorem 4 could be used to decrease $\lambda(r, s)$, contrary to hypothesis. Hence, every integer $1,2, \cdots, n$ occurs in $(r ; s)$ so that $\lambda(r)+\lambda(s)-\lambda(r, s)$ $=n$. Let $\lambda(r)=\rho$ so that $1 \leqq \rho<n$, and, by an application of Theorem 3 , let us cause all the integers $1,2, \cdots, \rho$ to be the ones occurring in $r$. Then, since $\rho+1, \rho+2, \cdots, n$ do not occur in $r$, these, as well as 
at least one of the numbers $1,2, \cdots, \rho$ must occur in $s$, since $(r ; s)$ was assumed improper.

Denote by $\alpha$ one of the indices common to $r$ and $s$. If, for some index $\beta$ in the range $\rho+1, \rho+2, \cdots, n$, the pair $\alpha, \beta$ were not blocked, then application of $[\beta \alpha]$ would reduce $\lambda(r, s)$ by one, contrary to hypothesis. Hence, every such pair $\alpha, \beta$ is blocked and, in particular, must be an associate pair since $\beta$ occurs in $s$ alone and the diagonal column is being disregarded. Consequently, since $\alpha$ must be an associate in $s$ of each of the indices $\rho+1, \rho+2, \cdots, n$, there must be at least $n-\rho$ repetitions in $r$. This is impossible since $\rho$ distinct integers plus $n-\rho$ repetitions cannot occur in $n-1$ columns.

9. Normal sets. We now fix our attention on proper, unitary dual-sets $(r ; s)$. Let us consider all nonsingular sets of the form $\left(r^{\prime} ; s^{\prime}\right)$ $=[\alpha \beta](r ; s)$; they are then necessarily proper and unitary. The proof of the Independence Theorem for $(r ; s)$ would, of course, follow from the proof for any one of the sets $\left(r^{\prime} ; s^{\prime}\right)$ (Theorem 4). We have seen in $\$ 8$ that the number $\lambda\left(r^{\prime}\right)+\lambda\left(s^{\prime}\right)$ cannot exceed $\lambda(r)+\lambda(s)$. If, for some pair of indices $\alpha, \beta$ we have $\lambda\left(r^{\prime}\right)+\lambda\left(s^{\prime}\right)<\lambda(r)+\lambda(s)$, let us then exclude $(r ; s)$ from further consideration and restrict our attention to $\left(r^{\prime} ; s^{\prime}\right)$. This process of reducing the number of dual-sets to be considered is brought to a halt by the requirement that all of the dual-sets be nonsingular.

These considerations lead us to the following definition. A proper, unitary set $(r ; s)$ is said to be irreducible if each $\left(r^{\prime} ; s^{\prime}\right)$ obtained from it by applications of the reduction Theorems, with the restriction $\lambda\left(r^{\prime}\right)$ $+\lambda\left(s^{\prime}\right)<\lambda(r)+\lambda(s)$, is singular. All other proper, unitary sets are said to be reducible.

It is clear that if a given pair of indices $\alpha, \beta$, both occurring in the same row of a dual-set, is not a blocked pair, then the dual-set is reducible. Hence, every pair of indices occurring in the same row of an irreducible dual-set must be a blocked pair, and, in view of our usual agreement on the diagonal column, must be associates in that row. Thus the application of the reduction Theorem 4 can no longer aid us in restricting the class of dual-sets for which the Independence Theorem need be proved ${ }^{3}$.

We still have available the reduction Theorems $1,2,3$, and 5 which enable us to reorder columns, interchange rows, and rename indices. By the use of these theorems we may restrict our attention, finally, to the normal sets which we now describe.

(1) The diagonal column is the first column and $r_{1}=s_{1}=1$.

${ }^{3}$ If $\alpha$ occurs in the first row of the proper set $(r ; s)$ and $\beta$ in the second row, then $[\beta \alpha](r ; \mathrm{s})=(r ; s)$ while $[\alpha \beta](r ; s)=(\alpha \beta)(r ; s)$ so that Theorem 3 may be used to obtain the same result. 
(2) If $\lambda(r)=\rho, \lambda(r)+\lambda(s)=\sigma \leqq n$, let the distinct indices which occur in the first row be named $1,2, \cdots, \rho$ and those in the second row, exclusive of the diagonal column, be named $\rho+1, \rho+2$, $\cdots, \sigma\left(1 \leqq r_{i} \leqq \rho, \rho+1 \leqq s_{i} \leqq \sigma\right.$ except $\left.s_{1}=1\right)$.

(3) The columns are so ordered that for $2 \leqq i \leqq \sigma$ one of $r_{i j}, s_{i}$ occurs at least once in the preceding $i-1$ columns and the other does not, while for $i>\sigma$ both $r_{i}, s_{i}$ occur in the preceding $i-1$ columns.

(4) The set $(r ; s)$ is irreducible.

The restrictions in (1) and (2) may be deduced by the use of reduction Theorems 2 and 5 in the one case, and 3, in the other. The possibility of imposing the restriction (3) is not so obvious, although the only reduction Theorem involved is that which reorders columns. One way of doing this is as follows.

Immediately to the right of the diagonal column, place all of those columns for which $r_{i}=1$. Follow this by a column having 2 in the first row and a mate of 1 in the second row; then, by a column having 3 in the first row and a mate of 1 in the second row, etc., until we have placed a column having $\rho$ in the first row and a mate of 1 in the second row (all of these columns must necessarily appear in $(r ; s)$ since, by the property of irreducibility, 1 is associated with each of the numbers $2,3, \cdots, \rho)$. If every index $\rho+1, \rho+2, \cdots, \sigma$ is a mate of 1 , we have already put in place exactly $\sigma$ columns, and the remaining $n-\sigma$ columns may be made to follow these in any desired order. If, however, some integer in the range $\rho+1, \rho+2, \cdots, \sigma$ is not a mate of 1 , place in position, next, a single column chosen from the remaining unplaced columns and involving this integer. Doing this for all such integers, we find that we have now placed $\sigma$ columns and the remaining $n-\sigma$ columns may again be placed as desired. It is easy to verify that this set will satisfy the requirement (3).

10. Proof of the Independence Theorem for normal sets. We shall show that, corresponding to every normal set $(r ; s)$, we can exhibit a matrix $A$ for which $V_{s}^{r}(A) \neq 0$.

Let

$$
A_{0}=\sum_{i=1}^{n} \lambda_{i} E_{i i}, \quad B_{0}=\sum_{j=2}^{n} \mu_{j} E_{r_{j} s_{j}},
$$

where the $\lambda_{i}$ and $\mu_{\imath}$ are indeterminates and $E_{i j}$ is the $n$th order square matrix having all elements equal to zero except the element in the $i$ th row and $j$ th column, which is unity. Since the matrix $A_{0}$ is a diagonal matrix, we have $A_{0}^{k}=\sum_{i=1}^{n} \lambda_{i}^{k} E_{i i}$ and 


$$
A_{0}^{k} E_{u v}=\lambda_{u}^{k} E_{u v}, \quad E_{u v} A_{0}^{k}=\lambda_{v}^{k} E_{u v}, \quad E_{r_{j} s_{j}} B_{v}=B_{0} E_{r_{j} s_{j}}=0,
$$

where $u, v=1,2, \cdots, n$ and $j=2,3, \cdots, n$. The equations (3) depend, of course, on the hypothesis that $(r ; s)$ is normal (and hence, proper) so that no $r_{i}$ is equal to an $s_{j}$ except possibly for $j=1$ (which is not involved in $B_{0}$ ).

With the help of (3) we may verify by induction that

$$
\left(A_{0}+B_{0}\right)^{k}=A_{0}^{k}+\sum_{j=2}^{n} \mu_{j} \varphi_{k}\left(\lambda_{r_{j}}, \lambda_{s_{j}}\right) E_{r_{j} s_{j}},
$$

where

$$
\varphi_{k}(x, y)=\frac{x^{k}-y^{k}}{x-y}
$$

holds for every integer $k \geqq 0$.

Now, if we choose $\mu_{j}=\lambda_{r_{j}}-\lambda_{s_{j}}$ we see that:

$$
V_{s}^{r}\left(A_{0}+B_{0}\right)=\left|\lambda_{1}^{i-1}, \lambda_{r_{2}}^{i-1}-\lambda_{s_{2}}^{i-1}, \lambda_{r_{3}}^{i-1}-\lambda_{s_{3}}^{i-1}, \cdots, \lambda_{n_{n}}^{i-1}-\lambda_{s_{n}}^{i-1}\right|,
$$

where we have exhibited the $i$ th row of the determinant. Bearing in mind the requirement (3) for a normal set, we see that by elementary transformations of this determinant the first $\sigma$ columns can be made equal to

$$
\lambda_{1}^{i-1}, \lambda_{2}^{i-1}, \lambda_{3}^{i-1}, \cdots, \lambda_{\sigma}^{i-1}
$$

while the last $n-\sigma$ columns will be linear combinations of these. Thus we see that in the case $\sigma=n$ the determinant $V_{s}^{r}\left(A_{0}+B_{0}\right)$ is, except possibly for sign, equal to the classical determinant of Vandermonde, and therefore is not identically zero.

In order to complete the proof of the Independence Theorem for those normal sets $(r ; s)$ for which $\sigma<n$, consider the matrix

$$
C_{0}=\sum_{j=\sigma+1}^{n} \nu_{j}\left(E_{r_{j} j}+E_{j s_{j}}\right) \text {. }
$$

We prove by induction that

$$
\left.\left(A_{0}+B_{0}+C_{0}\right)^{k}=\left(A_{0}+B_{0}\right)^{k}+\sum_{j=\sigma+1}^{n} \varphi_{i k} E_{r_{j} j}+\psi_{j k} E_{j s_{j}}+\chi_{j k} E_{r_{j} s_{j}}\right),
$$

where

$$
\begin{gathered}
\varphi_{j k}=\nu_{j} \varphi_{k}\left(\lambda_{r_{j}}, \lambda_{j}\right), \quad \psi_{j k}=\nu_{j} \varphi_{k}\left(\lambda_{s_{j}}, \lambda_{j}\right), \\
\chi_{j k}=\nu_{j}^{2} \frac{\varphi_{k}\left(\lambda_{r_{j}}, \lambda_{s_{j}}\right)-\varphi_{k}\left(\lambda_{j}, \lambda_{s_{j}}\right)}{\lambda_{r_{j}}-\lambda_{j}} .
\end{gathered}
$$


Now, using (4) we may write

$$
\left(A_{0}+B_{0}+C_{0}\right)^{k}=A_{0}^{k}+\sum_{j=\sigma+1}^{n}\left(\varphi_{j k} E_{r_{j}}+\psi_{j k} E_{j s_{j}}\right)+P_{k}+Q_{k}
$$

where

$$
\begin{gathered}
P_{k}=\sum_{i=2}^{\sigma} \mu_{i} \varphi_{k}\left(\lambda_{r_{i}}, \lambda_{s_{i}}\right) E_{r_{i} s_{i}}, \\
Q_{k}=\sum_{j=1+\sigma}^{n}\left\{\frac{\nu_{j}^{2} \varphi_{k}\left(\lambda_{j}, \lambda_{s_{j}}\right)+\left[\mu_{j}\left(\lambda_{j}-\lambda_{r_{j}}\right)-\nu_{j}^{2}\right] \varphi_{k}\left(\lambda_{r_{j}}, \lambda_{s_{j}}\right)}{\lambda_{j}-\lambda_{r_{j}}}\right\} E_{r_{j} s_{j}} .
\end{gathered}
$$

In this case $(\sigma<n)$ we choose

$$
\begin{array}{cr}
\mu_{i}=\lambda_{r_{i}}-\lambda_{s_{i}} & (i=2,3, \cdots, \sigma) \\
\mu_{j}=\lambda_{j}-\lambda_{r_{j}}, \quad \nu_{j}^{2}\left(\lambda_{j}-\lambda_{r_{j}}\right)\left(\lambda_{j}-\lambda_{s_{j}}\right) & (j=\sigma+1, \cdots, n),
\end{array}
$$

and find that

$$
P_{h}=\sum_{i=2}^{\sigma}\left(\lambda_{r_{i}}^{k}-\lambda_{s_{i}}^{k}\right) E_{r_{i} s_{i}}, \quad Q_{h}=\sum_{j=1+\sigma}^{n}\left(\lambda_{j}^{k}-\lambda_{r_{j}}^{k}\right) E_{r_{j} s_{j}} .
$$

Using these expressions in (5) we obtain:

$$
\begin{aligned}
V_{s}^{r}\left(A_{0}+B_{0}+C_{0}\right) & =\left|\lambda_{i}^{i-1}, \lambda_{r_{2}}^{i-1}-\lambda_{s_{2}}^{i-1}, \cdots, \lambda_{r_{\sigma}}^{i-1}-\lambda_{s_{\sigma}}^{i-1}, \lambda_{\sigma+1}^{i-1}-\lambda_{r_{\sigma+1}}^{i-1}, \lambda_{n}^{i-1}-\lambda_{r_{n}}^{i-1}\right| \\
& = \pm\left|\lambda_{1}^{i-1}, \lambda_{2}^{i-1}, \cdots, \lambda_{n}^{i-1}\right| .
\end{aligned}
$$

The last reduction involves once again the use of the elementary transformations on determinants and the normalization which we have effected on $(r ; s)$, specifically the restriction $(3)$ in $\S 9$.

This completes the proof of the Independence Theorem for all nonsingular dual-sets; for, as we have shown previously if $V_{s}^{r}(A)$ vanishes identically, then there would exist a normal set for which this generalized determinant of Vandermonde would vanish identically.

\section{REFERENCES}

1. I. A. Barnett and H. Reingold, Invariants of a system of linear homogeneous differential equations of the second order, Duke Math. J., 6 (1940), 141-7.

2. I. A. Barnett and C. W. Mendel, Generalized determinants of Vandermonde, Math. Z., 52 (1950), 723-734.

UNIVERSITY OF ILLINOIS

UNIVERSITY OF CINCINNATI 


\section{PACIFIC JOURNAL OF MATHEMATICS}

\section{EDITORS}

H. L. Royden

Stanford University

Stanford, California

E. Hewits

University of Washington

Seattle 5 , Washington
R. P. Dilworth

California Institute of Technology Pasadena 4, California

E. G. Straus

University of California

Los Angeles 24, California

\section{ASSOCIATE EDITORS}
E. F. BECKENBACH
C. E. BURGESS
H. BUSEMANN
H. FEDERER

\author{
M. HALL \\ P. R. HALMOS \\ V. GANAPATHY IYER \\ R. D. JAMES
}

\author{
M. S. KNEBELMAN \\ I. NIVEN \\ T. G. OSTROM \\ M. M. SCHIFFER
}

\section{J. J. STOKER \\ G. SZEKERES \\ F. WOLF \\ K. YOSIDA}

\section{SUPPORTING INSTITUTIONS}

UNIVERSITY OF BRITISH COLUMBIA CALIFORNIA INSTITUTE OF TECHNOLOGY

UNIVERSITY OF CALIFORNIA

MONTANA STATE UNIVERSITY

UNIVERSITY OF NEVADA

OREGON STATE COLLEGE

UNIVERSITY OF OREGON

UNIVERSITY OF SOUTHERN CALIFORNIA
STANFORD UNIVERSITY

UNIVERSITY OF UTAH

WASHINGTON STATE COLLEGE

UNIVERSITY OF WASHINGTON

$*$ * * *

AMERICAN MATHEMATICAL SOCIETY

CALIFORNIA RESEARCH CORPORATION HUGHES AIRCRAFT COMPANY

Mathematical papers intended for publication in the Pacific Journal of Mathematics should be typewritten (double spaced), and the author should keep a complete copy. Manuscripts may be sent to any of the editors. Manuscripts intended for the outgoing editors should be sent to their successors. All other communications to the editors should be addressed to the managing editor, E. G. Straus at the University of California, Los Angeles 24, California.

50 reprints of each article are furnished free of charge; additional copies may be obtained at cost in multiples of 50 .

The Pacific Journal of Mathematics is published quarterly, in March, June, September, and December. The price per volume (4 numbers) is $\$ 12.00$; single issues, $\$ 3.50$. Back numbers are available. Special price to individual faculty members of supporting institutions and to individual members of the American Mathematical Society: $\$ 4.00$ per volume; single issues, $\$ 1.25$.

Subscriptions, orders for back numbers, and changes of address should be sent to Pacific Journal of Mathematics, 2120 Oxford Street, Berkeley 4, California.

Printed at Kokusai Bunken Insatsusha (International Academic Printing Co., Ltd.), No. 10, 1-chome, Fujimi-cho, Chiyoda-ku, Tokyo, Japan.

\section{PUBLISHED BY PACIFIC JOURNAL OF MATHEMATICS, A NON-PROFIT CORPORATION}

The Supporting Institutions listed above contribute to the cost of publication of this Journal, but they are not owners or publishers and have no responsibility for its content or policies. 


\section{Pacific Journal of Mathematics}

\section{Vol. 6, No. 4}

1956

Seymour Ginsburg, On mappings from the family of well ordered subsets of

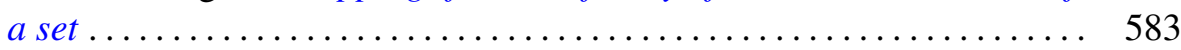

Leon Ehrenpreis, Some properties of distributions on Lie groups ......... 591

Marion K. Fort, Jr., A geometric problem of Sherman Stein ............. 607

Paul R. Garabedian, Calculation of axially symmetric cavities and jets . . . . 611

Walter Mossman Gilbert, Completely monotonic functions on cones ...... 685

William L. Hart and T. S. Motzkin, A composite Newton-Raphson gradient method for the solution of systems of equations ................. 691

C. W. Mendel and I. A. Barnett, A functional independence theorem for square matrices ................................. 709

Howard Ashley Osborn, The problem of continuous programs .......... 721

William T. Reid, Oscillation criteria for linear differential systems with complex coefficients ............................. 733

Irma Reiner, On the two-adic density of representations by quadratic

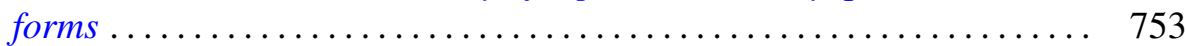

Shoichiro Sakai, A characterization of $W^{*}$-algebras .............. 763

Robert Steinberg, Note on a theorem of Hadwiger................. 775

$\mathrm{J}$. Eldon Whitesitt, Construction of the lattice of complemented ideals within

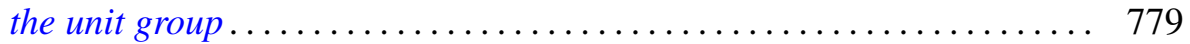

Paul Civin, Correction to "Some ergodic theorems involving two operators"... 